\title{
NEIGHBORHOOD CONNECTED EDGE DOMINATION IN GRAPHS
}

\author{
C. SIVAGNANAM, M. P. KULANDAIVEL AND P. SELVARAJU
}

\begin{abstract}
Let $G=(V, E)$ be a connected graph. An edge dominating set $X$ of $G$ is called a neighborhood connected edge dominating set (nced-set) if the edge induced subgraph $<N(X)>$ is connected. The minimum cardinality of a nced-set of $G$ is called the neighborhood connected edge domination number of $G$ and is denoted by $\gamma_{n c}^{\prime}(G)$. In this paper, we initiate a study of this parameter.
\end{abstract}

\section{Introduction}

The graph $G=(V, E)$ we mean a finite, undirected and connected graph with neither loops nor multiple edges. The order and size of $G$ are denoted by $n$ and $m$ respectively. For graph theoretic terminology we refer to Chartrand and Lesniak [3] and Haynes et.al [4].

For any $v \in V$. The open neighborhood and the closed neighborhood of $v$ are denoted by $N(\nu)$ and $N[v]=N(\nu) \cup\{v\}$ respectively. If $S \subseteq V$, then $N(S)=\bigcup_{\nu \in S} N(\nu)$ and $N[S]=N(S) \cup S$. If $S \subseteq V$ and $u \in S$, then the private neighbor set of $u$ with respect to $S$ is defined by pn $[u, S]=$ $\{v: N[v] \cap S=\{u\}\}$. For any $e \in E$, the open neighborhood and the closed neighborhood of $e$ are denoted by $N(e)$ and $N[e]=N(e) \cup\{e\}$ respectively. If $X \subseteq E$, then $N(X)=\bigcup_{e \in X} N(e)$ and $N[X]=N(X) \cup X$. If $X \subseteq E$ and $e_{1} \in X$ then the private neighbor of $e_{1}$ with respect to $X$ is defined by $\operatorname{pn}\left[e_{1}, X\right]=\left\{e_{2}: N\left(e_{2}\right) \cap X=\left\{e_{1}\right\}\right\}$. The degree of an edge $e=u v$ of $G$ is defined by $\operatorname{deg} e=\operatorname{deg} u+\operatorname{deg} v-2 . \delta^{\prime}(G)\left(\Delta^{\prime}(G)\right)$ is the minimum (maximum) degree among the edges of $G$. Let $X \subseteq E$, a graph $G-X$ is obtained from the graph $G$ by removing the edges of $X$. Let $H$ be a subgraph of $G$ and let $e \in G, d(e, H)$ denotes the distance from $e$ to $H$.

A subset $S$ of $V$ is called a dominating set of $G$ if $N[S]=V$. The minimum(maximum) cardinality of a minimal dominating set of $G$ is called the domination number(upper domination number) of $G$ and is denoted by $\gamma(G)(\Gamma(G))$. An excellent treatment of the fundamentals of domination is given in the book by Haynes et.al [4]. A survey of several advanced topics in domination is given in the book edited by Haynes et.al [5].

Corresponding author: M. P. Kulandaivel.

2010 Mathematics Subject Classification. 05C70.

Key words and phrases. Neighborhood connected domination, neighborhood connected edge domination. 
S. Arumugam and C. Sivagnanam [1] introduced the concept of neighborhood connected domination. A dominating set $S$ of a connected graph $G$ is called a neighborhood connected dominating set (ncd-set) if the induced subgraph $\langle N(S)\rangle$ is connected. The minimum cardinality of a ncd-set of $G$ is called the neighborhood connected domination number of $G$ and is denoted by $\gamma_{n c}(G)$.

As an analogy to vertex domination, the concept of edge domination was introduced by Mitchell and Hedetniemi [7]. A set $X \subseteq E$ is said to be an edge dominating set if every edge in $E-X$ is adjacent to some edge in $X$. The edge domination number of $G$ is the cardinality of a smallest edge dominating set of $G$ and is denoted by $\gamma^{\prime}(G)$. S. Arumugam and S. Velammal [2] introduced the concept of connected edge domination of a connected graph. An edge dominating set $X$ of a connected graph $G$ is called a connected edge dominating set if the edge induced subgraph $\langle X\rangle$ is connected. The minimum cardinality of a connected edge dominating set of $G$ is called connected edge domination number and is denoted by $\gamma_{c}^{\prime}(G)$.

In this paper we study the edge analogue of neighborhood connected domination number. We need the following theorem.

Theorem 1.1. [1] For any graph $G, \gamma_{n c}(G) \leq\left\lceil\frac{n}{2}\right\rceil$.

\section{Main Results}

Definition 2.1. An edge dominating set $X$ of a connected graph $G$ is called the neighborhood connected edge dominating set (nced-set) if the edge induced subgraph $\langle N(X)\rangle$ of $G$ is connected. The minimum cardinality of a nced-set is called the neighborhood connected edge domination number (nced-number) and is denoted by $\gamma_{n c}^{\prime}(G)$.

Example. Consider the following graph $G$

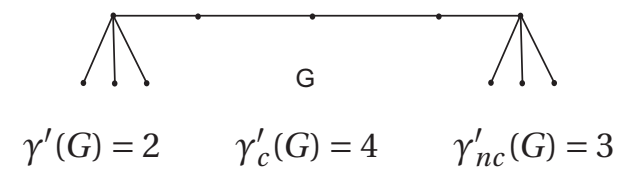

Remark 2.2. (i) Clearly $\gamma_{n c}^{\prime}(G) \geq \gamma^{\prime}(G)$. Further if $X$ is a connected edge dominating set with $|X|>1$ then $N(X)=E$ and hence $\gamma_{n c}^{\prime}(G) \leq \gamma_{c}^{\prime}(G)$.

(ii) For any connected graph $G$ that is not a star $\gamma_{n c}^{\prime}(G)=1$ if and only if there exists a non cut edge $e$ such that deg $e=m-1$. That is $G$ contains two adjacent vertices $u$ and $v$ such that all other vertices are mutually non adjacent, adjacent to either $u$ or $v$, and at least one vertex is adjacent to both $u$ and $v$. 
Theorem 2.3. For any graph $G, \gamma^{\prime}(G) \leq \gamma_{n c}^{\prime}(G) \leq 2 \gamma^{\prime}(G)$. Further given two positive integers a and $b$ with $a \leq b \leq 2 a$, there exists a graph $G$ with $\gamma^{\prime}(G)=a$ and $\gamma_{n c}^{\prime}(G)=b$.

Proof. Let $G$ be a connected graph and let $X$ be an edge dominating set of $G$. Obviously pairing each $e \in X$ with a private neighbor forms a nced-set of cardinality $2 \gamma^{\prime}(G)$.

Now, let $a$ and $b$ be two positive integers with $a \leq b \leq 2 a$. Let $b=a+k, 0 \leq k \leq a$. Consider the galaxy with stars $G_{1}, G_{2}, \ldots, G_{a}$ with $\left|V\left(G_{i}\right)\right| \geq 3, \quad 1 \leq i \leq a$. Join the maximum degree vertices of $G_{i}$ and $G_{i+1}$ by an edge $e_{i}, 1 \leq i \leq a$. Let $H$ be the graph obtained from the above graph by subdividing exactly once the edges $e_{i}$ where $1 \leq i \leq a-1$. Clearly $\gamma^{\prime}(H)=\gamma_{n c}^{\prime}(H)=a$. Let $G$ be the graph obtained from $H$ by subdividing an edge of $G_{i}$ exactly once where $1 \leq i \leq k$. Then $\gamma^{\prime}(G)=a$ and $\gamma_{n c}^{\prime}(G)=a+k=b$.

Theorem 2.4. For the path $P_{n}, n \geq 2, \gamma_{n c}^{\prime}\left(P_{n}\right)=\left\lceil\frac{n-1}{2}\right\rceil$.

Proof. Let $P_{n}=\left(v_{1}, v_{2}, \ldots, v_{n}\right)$ and let $e_{i}=v_{i} v_{i+1}$. If $n$ is odd, then $X=\left\{e_{j}: j=2 k\right.$ or $2 k+1$ and $k$ is odd $\}$ is a nced-set of $P_{n}$ and if $n$ is even then $X_{1}=X \cup\left\{e_{n-1}\right\}$ is a nced-set of $P_{n}$. Hence $\gamma_{n c}^{\prime}\left(P_{n}\right) \leq\left\lceil\frac{n-1}{2}\right\rceil$. Further, if $X$ is any $\gamma_{n c}^{\prime}$-set of $P_{n}$ then $N(X)$ contains all the internal edges of $P_{n}$ and hence $|X| \geq\left\lceil\frac{n-1}{2}\right\rceil$. Thus $\gamma_{n c}^{\prime}\left(P_{n}\right)=\left\lceil\frac{n-1}{2}\right\rceil$.

Corollary 2.5. For any non-trivial path $P_{n}$,

(i) $\gamma_{n c}^{\prime}\left(P_{n}\right)=\gamma^{\prime}\left(P_{n}\right)$ if and only if $n=3$ or 5 .

(ii) $\gamma_{n c}^{\prime}\left(P_{n}\right)=\gamma_{c}^{\prime}\left(P_{n}\right)$ if and only if $n=2,3,5$ or 6 .

Proof. Since $\gamma^{\prime}\left(P_{n}\right)=\left\lceil\frac{n-1}{3}\right\rceil$ and $\gamma_{c}^{\prime}\left(P_{n}\right)=n-3$ the corollary follows.

Theorem 2.6. For the cycle $C_{n}$ on $n$ vertices

$$
\gamma_{n c}^{\prime}\left(C_{n}\right)=\left\{\begin{array}{l}
\left\lceil\frac{n}{2}\right\rceil \text { if } n \neq 3(\bmod 4) \\
\left\lfloor\frac{n}{2}\right\rfloor \text { if } n \equiv 3(\bmod 4)
\end{array}\right.
$$

Proof. Let $C_{n}=\left(v_{1}, v_{2}, \ldots, v_{n}, v_{1}\right)$ and $n=4 k+r$ where $0 \leq r \leq 3$ and $e_{i}=v_{i} v_{i+1}$. Let $X=\left\{e_{i}\right.$ : $i=2 j, 2 j+1, j$ is odd and $1 \leq j \leq 2 k-1\}$.

$$
\text { Let } X_{1}= \begin{cases}X & \text { if } n \equiv 0(\bmod 4) \\ X \cup\left\{e_{n}\right\} & \text { if } n \equiv 1 \operatorname{or} 2(\bmod 4) \\ X \cup\left\{e_{n-1}\right\} & \text { if } n \equiv 3(\bmod 4)\end{cases}
$$


Clearly $X_{1}$ is a nced-set of $C_{n}$ and hence

$$
\gamma_{n c}^{\prime}\left(C_{n}\right) \leq\left\{\begin{array}{l}
\left\lceil\frac{n}{2}\right\rceil \text { if } n \neq 3(\bmod 4) \\
\left\lfloor\frac{n}{2}\right\rfloor \text { if } n \equiv 3(\bmod 4)
\end{array}\right.
$$

Now, let $X$ be any $\gamma_{n c}^{\prime}$-set of $C_{n}$ then $<X>$ contains at most one isolated edge and

$$
<N(X)>= \begin{cases}C_{n}-\{e\} & \text { if } n \not \equiv 0(\bmod 4) \\ C_{n} & \text { if } n \equiv 0(\bmod 4)\end{cases}
$$

Hence

$$
|X| \geq\left\{\begin{array}{l}
\left\lceil\frac{n}{2}\right\rceil \text { if } n \neq 3(\bmod 4) \\
\left\lfloor\frac{n}{2}\right\rfloor \text { if } n \equiv 3(\bmod 4)
\end{array}\right.
$$

and the result follows.

Corollary 2.7. (i) $\gamma_{n c}^{\prime}\left(C_{n}\right)=\gamma^{\prime}\left(C_{n}\right)$ if and only if $n=3,4$ or 7 .

(ii) $\gamma_{n c}^{\prime}\left(C_{n}\right)=\gamma_{c}^{\prime}\left(C_{n}\right)$ if and only if $n=3,4$ or 5 .

Proof. Since $\gamma^{\prime}\left(C_{n}\right)=\left\lceil\frac{n}{3}\right\rceil$ and $\gamma_{c}^{\prime}\left(C_{n}\right)=n-2$ the result follows.

Theorem 2.8. $\gamma_{n c}^{\prime}\left(K_{n}\right)=\left\lfloor\frac{n}{2}\right\rfloor, n \geq 3$.

Proof. Let $X$ be a maximum matching of $K_{n}$. Hence $X$ is an edge dominating set. Also $<N(X)>=K_{n}-X$ which is connected. Hence $X$ is a nced-set which implies $\gamma_{n c}^{\prime}\left(K_{n}\right) \leq|X|=$ $\left\lfloor\frac{n}{2}\right\rfloor$. Since $\gamma^{\prime}\left(K_{n}\right)=\left\lfloor\frac{n}{2}\right\rfloor$ the result follows.

Theorem 2.9. $\gamma_{n c}^{\prime}\left(K_{r, s}\right)=\min \{r, s\}$.

Proof. Let $v$ be a vertex such that $\operatorname{deg} v=\min \{r, s\}$. Let $X$ be the set of all edges incident with $v$. It is clear that $X$ is an edge dominating set. Also $\left\langle N(X)>=K_{r, s}\right.$, if $K_{r, s}$ is not a star and $<N(X)>=K_{1, n-1}$ if $K_{r, s}$ is a star. Thus $X$ is a nced-set. Hence $\gamma_{n c}^{\prime}\left(K_{r, s}\right) \leq|X|=\operatorname{deg} v=$ $\min \{r, s\}$. Since $\gamma^{\prime}\left(K_{r, s}\right)=\min \{r, s\}$ the result follows.

Theorem 2.10. For a tree $T, \gamma_{n c}^{\prime}(T)=1$ if and only if $T$ is a star.

Proof. Let $\gamma_{n c}^{\prime}(T)=1$ and let $X=\{e\}$ be the $\gamma_{n c}^{\prime}$-set of $G$. Let $e=u v$ and let $\operatorname{deg} u \geq 2$. If $\operatorname{deg} v>1$ then $\langle N(X)\rangle=T-e$ is disconnected. Hence $\operatorname{deg} v=1$. Thus $T$ is a star. The converse is obvious.

We now proceed to obtain a characterization of minimal nced-sets. 
Lemma 2.11. A superset of a nced-set is a nced-set.

Proof. Let $X$ be a nced-set of a graph $G$ and let $X_{1}=X \cup\{e\}$, where $e \in E-X$. Let $e=u v$. Clearly $e \in N(X)$ and $X_{1}$ is an edge dominating set of $G$. Now Let $x, y \in V\left(<N\left(X_{1}\right)>\right)$. If $x, y \in$ $V(<N(X)>)$ then any $x-y$ path in $<N(X)>$ is a $x-y$ path in $<N\left(X_{1}\right)>$. If $x \in V(<N(X)>)$ and $y \notin V(<N(X)>)$, then without loss of generality we assume $x-u$ path in $\langle N(X)\rangle$, and hence $x-u$ path together with $u-y$ path gives a $x-y$ path in $<N\left(X_{1}\right)>$. Also if $x, y \notin V(<$ $N(X)>)$ then $(x, u, v, y)$ or $(x, v, u, y)$ or $(x, u, y)$ or $(x, v, y)$ or $(x, y)$ is a $x-y$ path in $\left\langle N\left(X_{1}\right)>\right.$. Thus $<N\left(X_{1}\right)>$ is connected, so that $X_{1}$ is a nced-set of $G$.

Theorem 2.12. A nced-set $X$ of a graph $G$ is a minimal nced-set if and only if for every $e \in X$, one of the following holds,

(i) $\operatorname{pn}[e, X] \neq \varnothing$.

(ii) There exists two vertices $x, y \in<N(X)>$ such that every $x-y$ path in $\langle N(X)>$ contains at least one edge of $N(X)-N(X-\{e\})$.

Proof. Let $X$ be a minimal nced-set of $G$. Let $e \in X$ and let $X_{1}=X-\{e\}$. Then either $X_{1}$ is not an edge dominating set of $G$ or $\left\langle N\left(X_{1}\right)\right\rangle$ is disconnected. If $X_{1}$ is not an edge dominating set of $G$, then $\operatorname{pn}[e, X] \neq \varnothing$. If $<N\left(X_{1}\right)>$ is disconnected, then there exists two vertices $x, y \in<N\left(X_{1}\right)>$ such that there is no $x-y$ path in $\left\langle N\left(X_{1}\right)>\right.$. Since $\langle N(X)>$ is connected, it follows that every $x-y$ path in $\left\langle N\left(X_{1}\right)>\right.$ contains atleast one edge of $N(X)-$ $N(X-\{e\})$. Conversely, $X$ is a nced-set of $G$ satisfying the conditions of theorem, then $X$ is 1-minimal and hence the result follows from Lemma 2.11.

Theorem 2.13. Let $G$ be a graph with $\Delta^{\prime}=m-1$. Then $\gamma_{n c}^{\prime}(G)=1$ or 2 . Further $\gamma_{n c}^{\prime}(G)=2$ if and only if $G$ is a bistar, $B(r, s), r, s \geq 1$.

Proof. Let $e \in E(G)$ with deg $e=m-1$. Then $\left\{e, e_{1}\right\}$, where $e_{1} \in E-\{e\}$ is a nced-set of $G$ so that $\gamma_{n c}^{\prime}(G) \leq 2$. Now suppose $\gamma_{n c}^{\prime}(G)=2$. Then $<N(e)>=G-\{e\}$ is disconnected and hence $e$ is a cut edge of $G$. Let $e=u v$. Since dege $=m-1, N[u, v]-\{u, v\}$ is an independent set. If $\operatorname{deg} u$ or $\operatorname{deg} v$ is equal to 1 than $G$ is a star which is a contradiction to $\gamma_{n c}^{\prime}(G)=2$. Thus $\operatorname{deg} u \geq 2$ and $\operatorname{deg} v \geq 2$. Hence $G$ is a bistar $B(r, s), r, s \geq 1$. The converse is obvious.

In the following theorems we obtain a bound for $\gamma_{n c}^{\prime}(G)$.

Theorem 2.14. Let $G$ be a graph with $\Delta^{\prime}<m-1$. Then $\gamma_{n c}^{\prime}(G) \leq m-\Delta^{\prime}$.

Proof. Let $e \in E(G)$ and $\operatorname{deg} e=\Delta^{\prime}$. Since $G$ is connected and $\Delta^{\prime}<m-1$, there exists two adjacent edges $e_{1}$ and $e_{2}$ such that $e_{1} \in N(e)$ and $e_{2} \notin N[e]$. Now, let $X=\left(N(e)-\left\{e_{1}\right\}\right) \cup\left\{e_{2}\right\}$. Clearly $E-X$ is a nced-set of $G$ and hence $\gamma_{n c}^{\prime}(G) \leq m-\Delta^{\prime}$. 
Theorem 2.15. Let $T$ be a tree with $n>2$. Then $\gamma_{n c}^{\prime}(T)=m-\Delta^{\prime}$ if and only if $T$ is one of the following:

(i) Star.

(ii) Tree obtained from bistar $B\left(\left|X_{1}\right|,\left|X_{2}\right|\right)$ with $e=u v$ be a non-pendant edge and $X_{1}$ and $X_{2}$ are set of pendant edges which are incident with $u$ and $v$ respectively, by subdividing at least one edge of $X_{1} \cup X_{2}$ and subdividing at most one edge of $X_{1}$ or $X_{2}$ once, or by subdividing exactly one edge of $X_{1} \cup X_{2}$ twice.

Proof. Let $T$ be a tree with $\gamma_{n c}^{\prime}(T)=m-\Delta^{\prime}$. Let $e=u v \in E(T)$ and deg $e=\Delta^{\prime}$. Let $Y_{1}=N(u)-$ $\{v\}=\left\{v_{1}, v_{2}, \ldots, v_{r}\right\}$ and $Y_{2}=N(v)-\{u\}=\left\{v_{r+1}, v_{r+2}, \ldots, v_{\Delta^{\prime}}\right\}$. If $r=0$ then $T$ is a star graph. Let us assume $r \geq 1$ and $r<\Delta^{\prime}$ and $A=V(T)-N[u, v]=\left\{w_{1}, w_{2}, \ldots, w_{k}\right\}$ and $T_{1}=<A>$.

Case i. $E\left(T_{1}\right)=\varnothing$.

Suppose $\operatorname{deg} v_{i} \geq 3$ for some $v_{i} \in Y_{1} \cup Y_{2}$ without loss of generality we assume $v_{i} \in Y_{1}$. Let $u v_{i}, v_{i} w_{1}, v_{i} w_{2} \in E(T)$. Then $X=\left[E(T)-\left(N(e) \cup\left\{v_{i} w_{1}, v_{i} w_{2}\right\}\right)\right] \cup\left\{u v_{i}\right\}$ is a nced-set of $T$ and $|X|=m-\Delta^{\prime}-1$, which is a contradiction. Hence $\operatorname{deg} v_{i} \leq 2$. If $\operatorname{deg} v_{i}=1$ for all $i, \quad 1 \leq i \leq \Delta^{\prime}$ then $T$ is a bistar which is a contradiction. Thus $\operatorname{deg} v_{i}=2$ for some $i$.

Claim. At most one vertex of $Y_{1}$ or at most one vertex of $Y_{2}$ has degree 2.

Suppose $v_{1}, v_{2} \in Y_{1}$ and $v_{i}, v_{j} \in Y_{2}$ with $\operatorname{deg} v_{k}=2$, for $k \in\{1,2, i, j\}$. Let $w_{k} \in N\left(v_{k}\right)-$ $\{u, v\}$ for $k \in\{1,2, i, j\}$. Then $X=\left[E(T)-\left(N[e] \cup\left\{v_{1} w_{1}, v_{2} w_{2}, v_{i} w_{i}, v_{j} w_{j}\right\}\right)\right] \cup\left\{u v_{1}, u v_{2}, v v_{i}, v v_{j}\right\}$ is a nced-set with $|X|=m-\Delta^{\prime}-1$ which is a contradiction. Hence at most one vertex of $Y_{1}$ or at most one vertex of $Y_{2}$ has degree 2 .

Case ii. $\quad E\left(T_{1}\right) \neq \varnothing$.

Let $G_{1}$ be any non-trivial component of $T_{1}$ and we may assume without loss of generality that $v_{1} \in N\left[V\left(G_{1}\right)\right]$. If $G_{1}$ contains more than one pendant vertex of $T$, then $X=[E(T)-$ $\left.\left(N(e) \cup E_{1}\right)\right] \cup\left\{u v_{1}\right\}$ where $E_{1}$ is the set of all pendant edges of $T$ in $G_{1}$, is a nced-set of $T$ with $|X|<m-\Delta^{\prime}$ which is a contradiction. Hence $G_{1}$ is a path. Suppose $G_{1}=\left(x_{1}, x_{2}, \ldots, x_{k}\right), k \geq 3$ and let $v_{1} x_{1} \in E(T)$. Then $X=\left[E(T)-\left[N(e) \cup\left\{v_{1} x_{1}, x_{1} x_{2}\right\}\right]\right] \cup\left\{u v_{1}\right\}$ is a nced-set of $T$ with $|X|=$ $m-\Delta^{\prime}-1$ which is a contradiction. Thus $G_{1}=P_{2}$. Now, if $T$ has two non-trivial components $G_{1}=\left(x_{1}, x_{2}\right)$ and $G_{2}=\left(y_{1}, y_{2}\right), x_{1} \in N\left(v_{i}\right), y_{1} \in N\left(v_{j}\right)$ then $X=\left[E(T)-N(e) \cup\left\{v_{i} x_{1}, v_{j} y_{1}\right\}\right] \cup$ $\left\{u v_{i}\right\}$ is a nced-set of $T$ which is again a contradiction. Thus $T_{1}$ has exactly one non-trivial component. Let $X_{1}=\left\{u v_{i}: 1 \leq i \leq r\right\}$ and $X_{2}=\left\{v v_{j}: r+1 \leq j \leq \Delta^{\prime}\right\}$ then the result follows and the converse is obvious.

Theorem 2.16. Let $G$ be a unicyclic graph with cycle $C=\left(v_{1}, v_{2}, \ldots, v_{r}, v_{1}\right)$. Then $\gamma_{n c}^{\prime}(G)=$ $m-\Delta^{\prime}$ if and only if $G$ is isomorphic to $C_{3}$ or $C_{4}$ or $C_{5}$ or one of the graphs $G_{i}, 1 \leq i \leq 23$, given in Figure 1. 


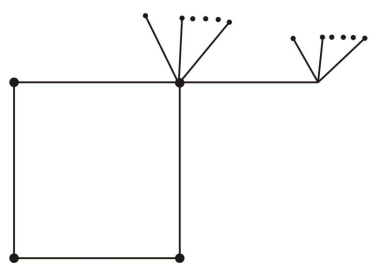

$\mathbf{G}_{1}$
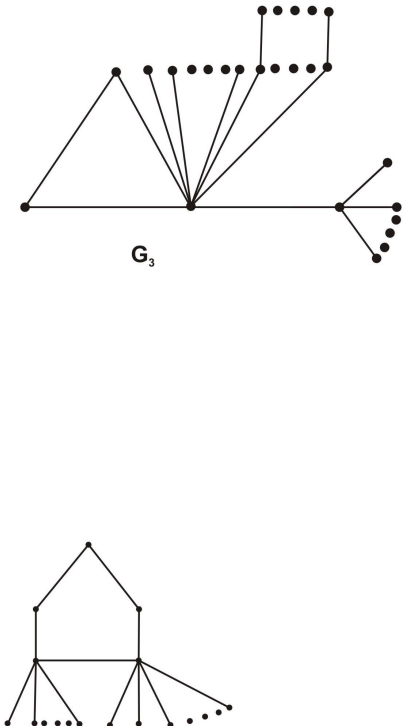

$\mathbf{G}_{5}$

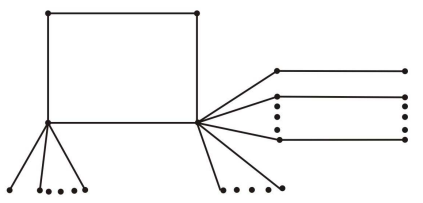

$\mathbf{G}_{8}$

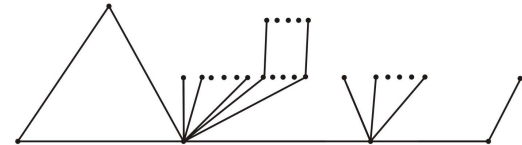

$\mathbf{G}_{2}$

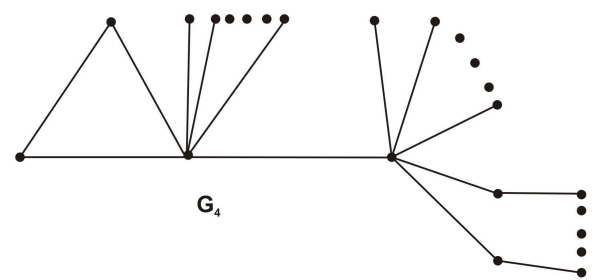

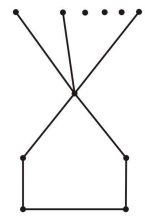

$\mathbf{G}_{6}$
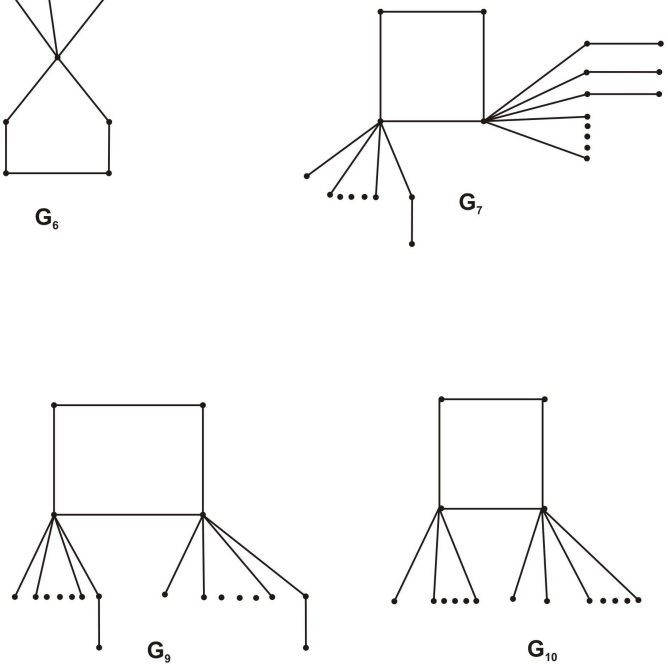


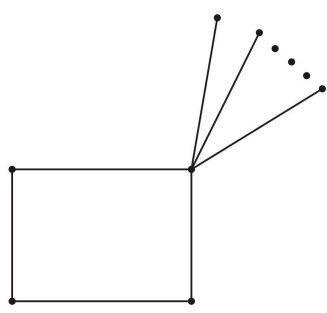

$\mathbf{G}_{11}$

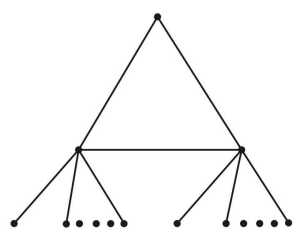

$\mathbf{G}_{14}$

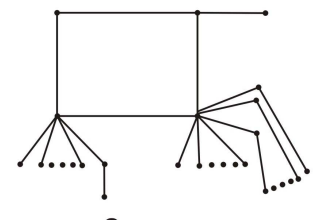

$\mathbf{G}_{17}$

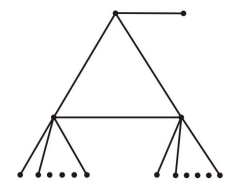

$\mathbf{G}_{20}$

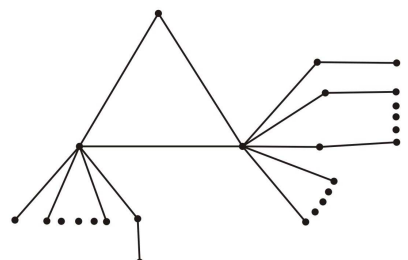

$\mathbf{G}_{12}$

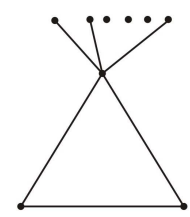

$\mathbf{G}_{15}$

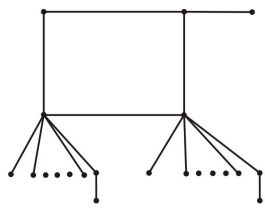

$\mathbf{G}_{18}$

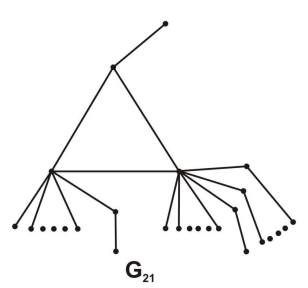

$\mathbf{G}_{21}$

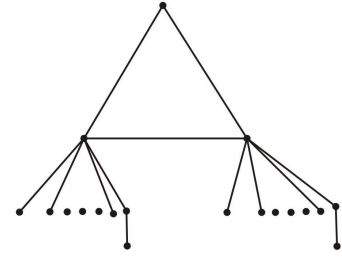

$\mathbf{G}_{13}$

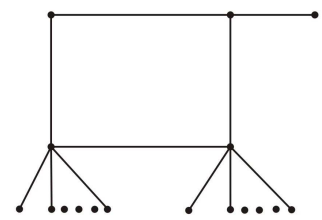

$\mathbf{G}_{16}$

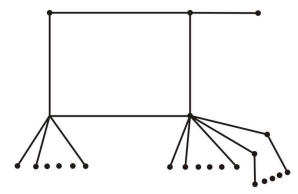

$\mathbf{G}_{19}$

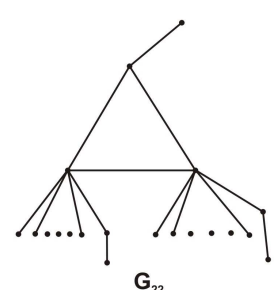

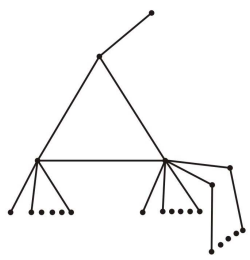

$\mathbf{G}_{23}$
Figure 1

Proof. Let $G$ be a unicyclic graph with cycle $C$ and $\gamma_{n c}^{\prime}(G)=m-\Delta^{\prime}$. If $G=C$ then it follows from theorem 2.6 that $m \leq 5$ and hence $G$ is isomorphic to $C_{3}$ or $C_{4}$ or $C_{5}$. Suppose $G \neq C$. Let $A$ denote the set of all pendant edges in $G$ and let $|A|=k$. Suppose $k \geq \Delta^{\prime}+1$. Since $E(G)-A$ 
is a nced-set of $G$ we have $\gamma_{n c}^{\prime}(G) \leq m-\Delta^{\prime}-1$ which is a contradiction. Hence $k \leq \Delta^{\prime}$. Also maximum of two adjacent edges of $e$ are in $C$ we have $\Delta^{\prime}-2 \leq k$.

Hence $\Delta^{\prime}-2 \leq k \leq \Delta^{\prime}$.

Let $e=u v$ with $\operatorname{deg} e=\Delta^{\prime}(G)$. Suppose $d(e, C) \geq 1$, then $k=\Delta^{\prime}$ or $\Delta^{\prime}-1$. Then $X=$ $[E(G)-E(C) \cup A] \cup X_{1}$ where $X_{1}$ is nced-set of $C$, is a nced-set of $G$ with $|X|<m-\Delta^{\prime}$ which is a contradiction. Hence the edge $e$ lies on $C$ or incident with $C$. Let $e$ be incident with $C$ and let $C=\left(v_{1}, v_{2}, \ldots, v_{r}, v_{1}\right)$. Let us assume $u=v_{1}$.

Claim. $r \leq 4$.

Suppose $r \geq 6$. Then any $\gamma_{n c}^{\prime}$-set of $C$ does not contain at least 3 edges of $C$. Let $X_{1}$ be a $\gamma_{n c}^{\prime}$-set of $C$ which contains an edge adjacent to $e$. Then $X=[E(G)-(E(C) \cup A)] \cup X_{1}$ is a nced-set of $G$ with $|X|<m-\Delta^{\prime}$ which is a contradiction. Hence $r \leq 5$. Suppose $r=5$. Let $C=\left(v_{1}, v_{2}, v_{3}, v_{4}, v_{5}, v_{1}\right)$. Then $X=E(G)-\left[A \cup\left\{v_{1} v_{2}, v_{2} v_{3}, v_{4} v_{5}\right\}\right]$ is a nced-set of $G$ with $|X|<m-\Delta^{\prime}$ which is a contradiction. Hence $r \leq 4$ and it is clear that every vertex in $V(C)-\left\{v_{1}\right\}$ has degree 2 .

Case 1.1. $r=4$.

Let $C=\left(v_{1}, v_{2}, v_{3}, v_{4}, v_{1}\right)$. Suppose there exists a vertex $w \in A$ such that $d(w, e) \geq 2$. Let $d(w, u)=d(w, e)$ and let $\left(u, w_{1}, w_{2} \ldots, w_{k}, w\right), k \geq 1$ be the unique $u-w$ path. Then $X=$ $\left[E(G)-\left[A \cup\left\{v_{1} v_{2}, v_{3} v_{4}, v_{4} v_{1}, w_{1} w_{2}\right\}\right]\right] \cup\left\{u w_{1}\right\}$ is a nced-set of $G$ with $|X|<m-\Delta^{\prime}$ which is a contradiction. Similarly we can get a contradiction if $d(w, v)=d(w, e)$. Hence $d(w, e)=1$ for all $w \in A$. Thus $G$ is isomorphic to $G_{1}$.

Case 1.2. $r=3$.

Let $C=\left(v_{1}, v_{2}, v_{3}, v_{1}\right)$ and $u=v_{1}$, suppose there exists a vertex $w \in A$ such that $d(w, e) \geq$ 3. Let $d(w, u)=d(w, e)$ and let $\left(u, w_{1}, w_{2}, \ldots, w_{k}, w\right), k \geq 2$ be the unique $u-w$ path. Then $X=\left[E(G)-\left[A \cup\left\{v_{2} v_{3}, v_{3} v_{1}, u w_{1}, w_{1} w_{2}\right\}\right]\right] \cup\left\{w_{k} w\right\}$ is a nced-set of $G$ with $|X|<m-\Delta^{\prime}$ which is a contradiction. Similarly we can get a contradiction if $d(w, v)=d(w, e)$. Hence $d(w, e) \leq 2$ for all $w \in A$. Let $w_{1} \in N(u)-[V(C) \cup\{v\}]$ and $\operatorname{deg} w_{1} \geq 3$. Then $X=E(G)-\left[A \cup\left\{v_{2} v_{3}, v_{3} \nu_{1}\right\}\right]$ is a nced-set of $G$ with $|X|<m-\Delta^{\prime}$ which is a contradiction. Similarly we can get a contradiction if $w_{1} \in N(v)-\{u\}$. Now, let $w_{1}, w_{2} \in N(u)-[V(C) \cup\{v\}]$ such that $\operatorname{deg} w_{1}=\operatorname{deg} w_{2}=2$. Suppose there exist two vertices $w_{3}, w_{4} \in N(v)-\{u\}$ such that $\operatorname{deg} w_{3}=\operatorname{deg} w_{4}=2$. Then $X=E(G)-[A \cup$ $\left.\left\{v_{2} v_{3}, v_{3} v_{1}, e\right\}\right]$ is a nced-set of $G$ with $|X|<m-\Delta^{\prime}$. Hence at most one vertex of $N(v)-\{u\}$ is of degree 2. Then $G$ is isomorphic to $G_{2}$ or $G_{3}$. Let $w_{1}, w_{2} \in N(v)-\{u\}$ with $\operatorname{deg} w_{1}=\operatorname{deg} w_{2}=2$. Suppose there exists a vertex $w_{3} \in N(u)-[V(C) \cup\{v\}]$ such that $\operatorname{deg} w_{3}=2$. Then $X=E(G)-$ $\left[A \cup\left\{v_{2} v_{3}, v_{3} v_{1}, e\right\}\right]$ is a nced-set of $G$ with $|X|<m-\Delta^{\prime}$ which is a contradiction. Hence $G$ is isomorphic to $G_{4}$.

Suppose $e$ lies on $C$. Let $C=\left(v_{1}, v_{2}, \ldots, v_{r}, v_{1}\right)$ and $v_{1} v_{2}=e$ 
Claim 1. $\operatorname{deg} w=1$ or 2 for all $w \in V(G)-V(C)$.

Suppose there exist a vertex $w \in V(G)-V(C)$ with $\operatorname{deg} w>2$. Then $k=\Delta^{\prime}-1$ or $\Delta^{\prime}$. If $k=\Delta^{\prime}-1$, then all the vertices of $V(C)-\left\{v_{1}, v_{2}\right\}$ have degree 2 and hence $X=E(G)-[A \cup$ $\left.\left\{v_{2} v_{3}, v_{2} v_{1}\right\}\right]$ is a nced-set of $G$ with $|X|<m-\Delta^{\prime}$. If $k=\Delta^{\prime}$ then $X=E(G)-\left[A \cup\left\{v_{2} v_{3}\right\}\right]$ is a ncedset of $G$ with $|X|<m-\Delta^{\prime}$ which is a contradiction. Hence deg $w=1$ or 2 for all $w \in V(G)-V(C)$.

Claim 2. Every vertex of $V(C)-\left\{v_{1}, v_{2}\right\}$ has degree 2 or 3 .

It follows from (1) that $\operatorname{deg} v_{i} \leq 4$ for all $i \neq 1,2$. If there exists a vertex $v_{i} \in V(C)$ with $\operatorname{deg} v_{i}=4$, then $k=\Delta^{\prime}$ and $X=E(G)-\left[A \cup\left\{v_{2} v_{3}\right\}\right]$ is a nced-set of $G$ with $|X|<m-\Delta^{\prime}$. This proves claim 2 .

Claim 3. $r \leq 5$.

Suppose $r \geq 6$. If $k=\Delta^{\prime}$ then $X=E(G)-\left[A \cup\left\{v_{2} v_{3}\right\}\right]$ is a nced-set of $G$ with $|X|<m-\Delta^{\prime}$. If $k=\Delta^{\prime}-1$ then there exists a vertex $v_{i}$ such that $\operatorname{deg} v_{i}=2$. Now $X=E(G)-\left[A \cup\left\{v_{i-1} v_{i}, v_{i} v_{i+1}\right\}\right]$ is a nced-set of $G$ with $|X|<m-\Delta^{\prime}$. If $k=\Delta^{\prime}-2$ then every vertex of $V(C)-\left\{v_{1}, v_{2}\right\}$ has degree 2 and hence $X=E(G)-\left[A \cup\left\{v_{2} v_{3}, v_{r-3} v_{r-2}, v_{r-2} v_{r-1}\right\}\right]$ is a nced-set of $G$ with $|X|<m-\Delta^{\prime}$. Thus $r \leq 5$.

Claim 4. $d(w, C) \leq 2$ for all $w \in A$.

Suppose there exist a pendant vertex $w_{1}$, such that $d\left(w_{1}, C\right) \geq 3$. Let $\left(w_{1}, w_{2}, \ldots, w_{k}, v_{i}\right), k \geq$ 3 be the unique $w_{1}-v_{i}$ path. If $k \neq \Delta-2$ then $X=\left[E(G)-\left[A \cup\left\{v_{2} v_{3}, v_{i} w_{k}, w_{k} w_{k-1}\right\}\right]\right] \cup\left\{w_{2} w_{1}\right\}$ is a nced-set of $G$ with $|X|<m-\Delta^{\prime}$. If $k=\Delta-2$, then $X=\left[E(G)-\left[A \cup\left\{v_{2} v_{3}, v_{3} v_{4}, v_{i} w_{k}, w_{k} w_{k-1}\right\}\right]\right] \cup$ $\left\{w_{2} w_{1}\right\}$ is a nced-set of $G$ with $|X|<m-\Delta^{\prime}$ which is a contradiction. Hence $d(w, C) \leq 2$ for all $w \in A$.

Claim 5. If there are two $P_{3}$ attached with $v_{1}$ then at most one $P_{3}$ is attached to $v_{2}$.

Suppose not, then $X=E(G)-\left[A \cup\left\{v_{1} v_{2}, v_{2} v_{3}, v_{r} v_{1}\right\}\right]$ is a nced-set of $G$ with $|X|<m-\Delta^{\prime}$ which is a contradiction. Hence the Claim 5.

Case 2.1. $k=\Delta^{\prime}-2$.

In this case $\operatorname{deg} x=1$ or 2 for all $x \in V(G)-\left\{\nu_{1}, v_{2}\right\}$. Now, if $r=5$ and if there exists a vertex $w \in N\left(v_{i}\right)-V(C), i=1$ or 2 , such that $\operatorname{deg} w=2$, then $X=E(G)-\left[A \cup\left\{v_{2} v_{3}, v_{3} v_{4}, v_{5} v_{1}\right\}\right]$ is a nced-set of $G$ with $|X|<m-\Delta^{\prime}$. Hence $\operatorname{deg} w=1$ for all $w \in N\left(\nu_{i}\right)-V(C)$ and hence $G$ is isomorphic to $G_{5}$ or $G_{6}$. If $r \leq 4$ then $G$ is isomorphic to $G_{i}, 7 \leq i \leq 15$.

Case 2.2. $k=\Delta^{\prime}-1$.

In this case $\operatorname{deg} v_{i}=3$ for exactly one vertex $v_{i} \neq v_{1}$ and $v_{2}$ on $C$ also $\operatorname{deg} x=1$ or 2 for all $x \in V(G)-\left\{v_{1}, v_{2}, v_{i}\right\}$. If $r=5$, then $X=E(G)-[A \cup B]$ where $B$ is a set of edges in $C$ not 
incident with $v_{i}$ is a nced-set of $G$ with $|X|=m-\Delta^{\prime}-1$ and hence $r=3$ or 4 . Suppose there exists a path $\left(v_{i}, x_{1}, w_{1}\right)$ such that $x_{1} \notin V(C)$ and $w_{2} \in A$, if $r=4$ then $X=[E(G)-[A \cup B \cup$ $\left.\left.\left\{v_{i} x_{1}\right\}\right]\right] \cup\left\{x_{1} w_{1}\right\}$ where $B$ is $N\left[v_{i} x_{1}\right] \cap V(C)$ is a nced-set of $G$ with $|X|<m-\Delta^{\prime}$ and if $r=3$, then $X=E(G)-\left[A \cup\left\{v_{2} v_{3}, v_{3} v_{1}, v_{3} x_{1}\right\}\right] \cup\left\{x_{1} w_{1}\right\}$ is a nced-set of $G$ with $|X|<m-\Delta^{\prime}$ and hence $G$ is isomorphic to $G_{i}, 16 \leq i \leq 23$.

Case 2.3. If $k=\Delta^{\prime}$.

In this case $r=4$ or 5 and there does not exists a graph with $\gamma_{n c}^{\prime}(G)=m-\Delta^{\prime}$. Converse is obvious.

Problem 2.17. Characterize the class of graphs for which $\gamma_{n c}^{\prime}(G)=m-\Delta^{\prime}$.

Remark 2.18. Since $\gamma_{n c}^{\prime}(G)=\gamma_{n c}(L(G))$ where $L(G)$ is the line graph of $G$, it follows from Theorem 1.1 that $\gamma_{n c}^{\prime}(G) \leq\left\lceil\frac{m}{2}\right\rceil$.

Theorem 2.19. Let $G$ be any graph such that both $G$ and $\bar{G}$ are connected. Then $\gamma_{n c}^{\prime}(G)+$ $\gamma_{n c}^{\prime}(\bar{G}) \leq m+1$.

Proof. The proof follows from Remark 2.18.

Remark 2.20. The bounds given in Theorem 2.19 is sharp. The graph $G=C_{5}, \gamma_{n c}^{\prime}(G)+\gamma_{n c}^{\prime}(\bar{G})=$ $6=m+1$.

Problem 2.21. Characterize the class of graphs for which $\gamma_{n c}^{\prime}(G)+\gamma_{n c}^{\prime}(\bar{G})=m+1$.

Theorem 2.22. For any graph $G, \gamma_{n c}^{\prime}(G) \leq\left\lfloor\frac{3 n}{4}\right\rfloor$.

Proof. Let $X$ be a maximum matching of the graph $G$. Label the edges of $X$ by $e_{1}, e_{2}, \ldots, e_{k}, e_{k+1}, \ldots, e_{r}$ such that the edges $e_{i}$ and $e_{i+1}, i$ is odd $1 \leq i \leq k-1$ are adjacent to common edge $f\left(e_{i}\right)$ with maximum value of $k$. Let $Y=\left\{f\left(e_{i}\right) / i\right.$ is odd $\}$. Then $X \cup Y$ is an edge dominating set with $<N(X \cup Y)>$ is connected and hence $\gamma_{n c}^{\prime}(G) \leq|X \cup Y|=\left\lfloor\frac{3 n}{4}\right\rfloor$.

Remark 2.23. The bound given in Theorem 2.22 is sharp. The graph $G=C_{5}, \gamma_{n c}^{\prime}(G)=3=$ $\left\lfloor\frac{3 n}{4}\right\rfloor$.

Problem 2.24. Characterize the class of graphs for which $\gamma_{n c}^{\prime}(G)=\left\lfloor\frac{3 n}{4}\right\rfloor$.

\section{Acknowledgement}

Thanks are due to the referees for their helpful comments. 


\section{References}

[1] S. Arumugam and C. Sivagnanam, Neighborhood connected domination in graphs, J. Combin. Math. Combin. Comput. 73(2010), 55-64.

[2] S. Arumugam and S. Velammal, Connected edge domination in graphs, Bulletin of Allahabad Mathematical Society, 24(1)(2009), 43-49.

[3] G. Chartrand and L. Lesniak, Graphs and Digraphs, CRC, (2005).

[4] T.W. Haynes, S.T. Hedetniemi and P.J. Slater, Fundamentals of Domination in Graphs, Marcel Dekker, Inc., New York, (1997).

[5] T.W. Haynes, S.T. Hedetniemi and P.J. Slater, Domination in Graphs-Advanced Topics, Marcel Dekker, Inc., New York, (1997).

[6] S.R. Jayaram, Line domination in graphs, Graphs Combin. 3 (1987), 357-363.

[7] S. Mitchell and S.T. Hedetniemi, Edge domination in trees, Congr. Numer. 19(1977), 489-509.

School of Sciences, Birla Institute of Technology, Kingdom of Bahrain.

E-mail: choshi71@gmail.com

Department of Mathematics, St. Joseph's College of Engineering, Chennai-600119, India.

E-mail: gracempk@yahoo.co.in

Department of Mathematics, SSN College of Engineering, Chennai-603110, India.

E-mail: pselvar@yahoo.com 